\title{
A dichotomy on Schreier sets
}

by

ROBERT JUDD (Stillwater, Okla.)

Abstract. We show that the Schreier sets $S_{\alpha}\left(\alpha<\omega_{1}\right)$ have the following dichotomy property. For every hereditary collection $\mathcal{F}$ of finite subsets of $\mathbb{N}$, either there exists infinite $M=\left(m_{i}\right)_{i=1}^{\infty} \subseteq \mathbb{N}$ such that $\mathcal{S}_{\alpha}(M)=\left\{\left\{m_{i}: i \in E\right\}: E \in \mathcal{S}_{\alpha}\right\} \subseteq \mathcal{F}$, or there exist infinite $M=\left(m_{i}\right)_{i=1}^{\infty}, N \subseteq \mathbb{N}$ such that $\mathcal{F}[N](M)=\left\{\left\{m_{i}: i \in F\right\}: F \in \mathcal{F}\right.$ and $\left.F \subset N\right\} \subseteq \mathcal{S}_{\alpha}$.

1. Introduction. Collections of finite subsets of the natural numbers have become important in Banach space theory. The Schreier sets $\mathcal{S}_{\alpha}$, defined below for each countable ordinal $\alpha$, are the most common among these sets. The first Schreier set, $\mathcal{S}_{1}$, is fundamental to the construction of the original Tsirelson space (see [T] and [FJ]), while the more general Schreier sets are used to construct the Schreier spaces, which may be found in [Sch], [AA] and $[\mathrm{AO}]$, and the exciting new collection of Tsirelson type spaces developed by Argyros and Deliyanni [AD]

The Banach spaces mentioned above may be constructed with collections of finite subsets of the natural numbers other than the Schreier sets. However, the Schreier sets are in some sense universal for these alternate collections. For example, a result of Odell, Tomczak and Wagner [OTW] shows that for pointwise closed collections $\mathcal{F}$ of finite subsets of $\mathbb{N}$ there exists a subsequence $N$ of $\mathbb{N}$ such that $\mathcal{F}(N)$ is a subset of one of the Schreier sets. (The notation $\mathcal{F}(N)$ is described below.)

We show roughly that if we fix a Schreier set $\mathcal{S}_{\alpha}$, then hereditary collections $\mathcal{F}$ of finite subsets of $\mathbb{N}$ satisfy: either $\mathcal{F}$ is of sufficient complexity to contain the Schreier set, or the sets in $\mathcal{F}$ lying in some subsequence must be contained in the Schreier set. The precise statement is a bit more complicated. One must allow for a wide range of collections of finite subsets. For example, the first Schreier set, $\mathcal{S}_{1}$, consists of all finite subsets of $\mathbb{N}$ whose smallest element is at least as large as the size of the set. This condition is called an admissibility condition. There are many such conditions. A different admissibility condition would be to consider collections of finite subsets

1991 Mathematics Subject Classification: Primary 46B20, 05D10. 
such that the square of the smallest element in each set is at least as large as the number of elements in the set. We state the dichotomy theorem here, deferring the notation until Section 2 .

THEOREM 1.1. For each $\alpha<\omega_{1}$, for every hereditary collection $\mathcal{F} \subseteq[\mathbb{N}]^{<\omega}$ and for all $\bar{M} \in[\mathbb{N}]$ either there exists $M \in[\bar{M}]$ such that $\mathcal{S}_{\alpha}(M) \subseteq \mathcal{F}$ or there exist $M \in[\bar{M}], N \in[\mathbb{N}]$ such that $\mathcal{F}[N](M) \subseteq \mathcal{S}_{\alpha}$.

In the next section we define the Schreier classes $\mathcal{S}_{\alpha}\left(\alpha<\omega_{1}\right)$ along with other notions concerning collections of finite subsets of $\mathbb{N}$. We also introduce Schreier games; these are a method of choosing finite subsets of $\mathbb{N}$ in such a way that the resulting set is in one of the collections $\mathcal{S}_{\alpha}$. The combinar torial framework for proving Theorem 1.1 is presented in Section 3 as the dichotomy property and the proof of Theorem 1.1 is given in Section 4 . We devote Section 5 to an alternative proof of a result of Argyros, Mercourakis and Tsarpalias [AMT], using Theorem 1.1.

2. Preliminaries. We use various subsets, and collections of subsets, of the natural numbers throughout this paper; for future reference we define all the notation for these sets at the beginning of this section. In general, $L$, $M$ and $N$ will be infinite subsets of $\mathbb{N}$, while $E$ and $F$ will be finite subsets, and $\mathcal{F}$ and $\mathcal{G}$ will be collections of finite subsets of $\mathbb{N}$. We consider every subset of $\mathbb{N}$, whether finite or infinite, to be an increasing sequence. Thus if $N \subseteq \mathbb{N}$, then $N=\left(n_{i}\right)_{i=1}^{\infty}$ where $n_{1}<n_{2}<\ldots$, and if $E$ is a finite subset, then $E=\left\{e_{1}, \ldots, e_{k}\right\}$ where $e_{1}<\ldots<e_{k}$.

When $N$ is an infinite subset of $\mathbb{N}$ we let $[N]$ be the set of infinite subsets of $N$ and we let $[N]^{<\omega}$ represent the set of finite subsets of $N$. Let $E, F \subseteq \mathbb{N}$ and $n \geq 1$. We write $E<F$ if either $F$ is empty or if $\max E<\min F, n<E$ if $\{n\}<E$, and $n \leq E$ if $n \leq \min E$.

Let $\mathcal{F}$ be a collection of finite subsets of $\mathbb{N}$. We next define three properties which $\mathcal{F}$ may have: hereditary, spreading and closed. For $\mathcal{F}$ to be hereditary requires that whenever $E \subset F$ and $F \in \mathcal{F}$ then $E \in \mathcal{F}$. We say $\mathcal{F}$ is spreading if whenever $F=\left\{m_{1}, \ldots, m_{k}\right\} \in \mathcal{F}$ and $n_{1}<\ldots<n_{k}$ satisfies $m_{i} \leq n_{i}$ for $i \leq k$, then $\left\{n_{1}, \ldots, n_{k}\right\} \in \mathcal{F}$. The set $2^{\mathbb{N}}$ of all subsets of $\mathbb{N}$ is a topological space under the topology of pointwise convergence; thus $\mathcal{F}$ is (pointwise) closed if it is closed in $2^{\mathbb{N}}$. We collect the first and third properties together and say that $\mathcal{F}$ is adequate if it is both closed and hereditary.

Finally we need some notation to talk about what happens when we restrict a collection of finite subsets of $\mathbb{N}$ to an infinite subset of $\mathbb{N}$. Let $N=\left(n_{i}\right) \in[\mathbb{N}]$ be an infinite sequence and let $\mathcal{F} \subseteq[\mathbb{N}]<\omega$ be a collection of finite subsets. We write the subset of $\mathcal{F}$ consisting only of those elements which are also subsets of $N$ as $\mathcal{F}[N]$. Thus

$$
\mathcal{F}[N]=\{F \in \mathcal{F}: F \subseteq N\}=\mathcal{F} \cap[N]^{<\omega} .
$$

We also want to put $\mathcal{F}$ into the sequence $N$. In other words, if $F \in \mathcal{F}$ and we define $n_{F}=\left\{n_{i}: i \in F\right\}$, then $\mathcal{F}(N)$ is the collection of all such sets, i.e. $\mathcal{F}(N)=\left\{n_{F}: F \in \mathcal{F}\right\}$. (Note that $n_{F}$ is a finite subset of $N$.)

DEFINITION 2.1. The Schreier sets, $\mathcal{S}_{\alpha}$ for each $\alpha<\omega_{1}$, are defined inductively as follows (see $[\mathrm{AA}]$ ): let $\mathcal{S}_{0}=\{\{n\}: n \geq 1\} \cup\{\emptyset\}$ and $\mathcal{S}_{1}=$ $\{F \subset \mathbb{N}:|F| \leq F\}$. (Note that this definition allows for $\emptyset \in \mathcal{S}_{1}$.) If $\mathcal{S}_{\alpha}$ has been defined then let

$$
\mathcal{S}_{\alpha+1}=\left\{\bigcup_{i=1}^{k} F_{i}: k \leq F_{1}<\ldots<F_{k}, F_{i} \in \mathcal{S}_{\alpha}(i=1, \ldots, k), k \in \mathbb{N}\right\} .
$$

If $\alpha$ is a limit ordinal with $\mathcal{S}_{\beta}$ defined for each $\beta<\alpha$, choose and fix an increasing sequence of ordinals $\left(\alpha_{n}\right)$ with $\alpha=\sup _{n} \alpha_{n}$ and let

$$
\mathcal{S}_{\alpha}=\bigcup_{n=1}^{\infty}\left\{F \in \mathcal{S}_{\alpha_{n}}: n \leq F\right\} \text {. }
$$

Note that each $\mathcal{S}_{\alpha}$ is hereditary, spreading and closed. The definition of $\mathcal{S}_{\alpha}$ for $\alpha$ infinite depends on choices made when selecting sequences increasing to limit ordinals. However, none of the results cited, nor obtained, in this paper depend on which particular sequences were used to construct the sets $\mathcal{S}_{\alpha}$. For $r \geq 1$ and $\alpha_{1}, \ldots, \alpha_{r}<\omega_{1}$ let

$$
\left(\mathcal{S}_{\alpha_{1}}, \ldots, \mathcal{S}_{\alpha_{r}}\right)=\left\{F=\bigcup_{i=1}^{r} F_{i}: F_{i} \in \mathcal{S}_{\alpha_{i}}(i \leq r) \text { and } F_{1}<\ldots<F_{r}\right\} \text {. }
$$

DEFINITION 2.2. We define a game for two players on $\mathbb{N}$, called an $\left(\alpha_{1}, \ldots, \alpha_{r}\right)$-Schreier game, for each $r$-tuple of ordinals with $0 \leq \alpha_{1} \leq \ldots \leq$ $\alpha_{r}<\omega_{1}$. If $r=1$, then we drop the parentheses and simply call it an $\alpha$-Schreier game. The two players are $\mathcal{N}$ who chooses numbers and $\mathcal{S}$ who chooses non-empty sets. Roughly, $\mathcal{N}$ will pick a finite sequence of numbers and $\mathcal{S}$ will pick a finite sequence $E_{1}<\ldots<E_{k}$ of finite subsets of $\mathbb{N}$. The number of choices made and the order of the plays will depend upon the particular $\left(\alpha_{1}, \ldots, \alpha_{r}\right)$-Schreier game being played, and may also depend upon previous plays.

We first describe the $\alpha$-Schreier game for $\alpha<\omega_{1}$. In the 0 -Schreier game $\mathcal{S}$ chooses $\{n\}$ for some $n \geq 1$. In the 1 -Schreier game $\mathcal{N}$ picks $l \geq 1$ and $\mathcal{S}$ chooses $E \in[\mathbb{N}]^{<\omega}$ such that $|E| \geq l$. Suppose we have already described the $\alpha$-Schreier game for $\alpha<\omega_{1}$. The $(\alpha+1)$-Schreier game starts with $\mathcal{N}$ picking $l \geq 1$ and then the two players play the $\alpha$-Schreier game $l$ times, with the additional condition that if $E$ is the last set $\mathcal{S}$ chose in the $i$ th $\alpha$-Schreier game and $F$ is the first set $\mathcal{S}$ chose in the $(i+1)$ th $\alpha$-Schreier game, then $E<F$. For $\alpha$ a limit ordinal suppose we have already described the $\gamma$-Schreier game for each $\gamma<\alpha$ and let $\alpha_{n} \nearrow \alpha$ be the sequence used to 
define $\mathcal{S}_{\alpha}$. The $\alpha$-Schreier game starts with $\mathcal{N}$ picking $l \geq 1$ and then the two players play the $\alpha_{l}$-Schreier game.

If $\alpha_{1} \leq \ldots \leq \alpha_{r}<\omega_{1}$, then an $\left(\alpha_{1}, \ldots, \alpha_{r}\right)$-Schreier game is simply an $\alpha_{1}$-Schreier game followed by an $\alpha_{2}$-Schreier game, and so on, finishing with an $\alpha_{r}$-Schreier game. The only other condition is that if $E$ is the last set $\mathcal{S}$ chose in the $\alpha_{i}$-Schreier game and $F$ is the first set $\mathcal{S}$ chose in the $\alpha_{i+1}$-Schreier game, then $E<F$. In the sequel, by an $\left(\alpha_{1}, \ldots, \alpha_{r}\right)$-game we shall mean an $\left(\alpha_{1}, \ldots, \alpha_{r}\right)$-Schreier game.

As an example, consider the 2-Schreier game. $\mathcal{N}$ chooses $l \geq 1$ and then they play the $(1, ., ., 1)$-game. This starts with $\mathcal{N}$ choosing $k_{1} \geq 1$ and then $\mathcal{S}$ chooses $E_{1}$ with $\left|E_{1}\right| \geq k_{1}$. Then $\mathcal{N}$ chooses $k_{2}$ and $\mathcal{S}$ chooses $E_{2}$ with $\left|E_{2}\right| \geq k_{2}$ and $E_{2}>E_{1}$. This continues until $\mathcal{N}$ has chosen $k_{l}$ and $\mathcal{S}$ has chosen $E_{l}$ with $\left|E_{l}\right| \geq k_{l}$ and $E_{l}>E_{l-1}$. The set resulting from this game is $E=\bigcup_{i=1}^{l} E_{i}$. In general, if $\mathcal{N}$ and $\mathcal{S}$ play a Schreier game, and $\left(E_{i}\right)_{i=1}^{k}$ is the sequence of sets which $\mathcal{S}$ chose in the game, with $E_{i}<E_{i+1}(1 \leq i<k)$, then the set $E$ resulting from the game is defined as $E=\bigcup_{i=1}^{k} E_{i}$.

A bound $\left(\alpha_{1}, \ldots, \alpha_{r}\right)$-Schreier game is one where at each stage $\mathcal{N}$ is restricted to exactly one choice of number to pick. If $\mathcal{N}$ and $\mathcal{S}$ play a bound game and $E$ is the set resulting from this game, then we say $\mathcal{S}$ chose $E$ as small as possible if at each stage, when $\mathcal{S}$ had to choose a set $E_{i}$ of size at least $l_{i}$, then $\mathcal{S}$ always chose $E_{i}$ of size equal to $l_{i}$.

We say that $\mathcal{N}$ has a winning strategy for the $\left(\alpha_{1}, \ldots, \alpha_{r}\right)$-Schreier game on $\mathcal{F} \subset[\mathbb{N}]<\omega$ if $\mathcal{N}$ can choose integers so that, whatever sets $\mathcal{S}$ picks, the set $E$ resulting from the game does not belong to $\mathcal{F}$. Notice that if $\mathcal{N}$ has a winning strategy for the $\left(\alpha_{1}, \ldots, \alpha_{r}\right)$-Schreier game on $\mathcal{F} \subseteq[\mathbb{N}]<\omega$, then $\mathcal{N}$ also has a winning strategy for the $\left(\alpha_{1}, \ldots, \alpha_{r}\right)$-Schreier game on $\mathcal{F}[M]$ for any $M \in[\mathbb{N}]$ since $\mathcal{F}[M] \subseteq \mathcal{F}$.

As an example of a winning strategy for $\mathcal{N}$ we shall consider the $(1,1)$ game on $\mathcal{S}_{1}$. In this game $\mathcal{N}$ chooses $l \geq 1$, next $\mathcal{S}$ chooses $E \in[\mathbb{N}]^{<\omega}$ with $|E| \geq l$, then $\mathcal{N}$ chooses $m \geq 1$ and finally $\mathcal{S}$ chooses $F>E$ with $|F| \geq m$. A winning strategy for $\mathcal{N}$ in this game would be to choose $l=1$ and $m=\min E$ (which $\mathcal{N}$ may do since $\mathcal{S}$ chooses $E$ before $\mathcal{N}$ chooses $m$ ). Now, if $A=E \cup F$, then $|A| \geq l+m=1+m>\min A$, while if $A$ were in $\mathcal{S}_{1}$, then we would have $|A| \leq \min A$. Thus $A \notin \mathcal{S}_{1}$, which is what $\mathcal{N}$ was trying to achieve.

\section{The Dichotomy Property}

Definition 3.1. An $r$-tuple of ordinals, $\left(\alpha_{1}, \ldots, \alpha_{r}\right)$ with $0 \leq \alpha_{1} \leq \ldots \leq$ $\alpha_{\tau}<\omega_{1}$, has the Dichotomy Property (D) if for each hereditary collection $\mathcal{F} \subseteq[\mathbb{N}]^{<\omega}$ and every $\bar{N} \in[\mathbb{N}]$, either there exists $M \in[\bar{N}]$ such that $\left(\mathcal{S}_{\alpha_{1}}, \ldots, \mathcal{S}_{\alpha_{r}}\right)(M) \subseteq \mathcal{F}$, or there exists $M \in[\bar{N}]$ such that $\mathcal{N}$ has a winning strategy for the $\left(\alpha_{1}, \ldots, \alpha_{r}\right)$-Schreier game on $\mathcal{F}[M]$.

This section is devoted to proving that every increasing $r$-tuple of countable ordinals has the Dichotomy Property.

Proposition 3.2. The r-tuple $\left(\alpha_{1}, \ldots, \alpha_{r}\right)$ has the Dichotomy Property (D) for each $r \geq 1$ and every $r$-tuple of ordinals with $0 \leq \alpha_{1} \leq \ldots \leq \alpha_{r}$ $<\omega_{1}$.

We prove this inductively in several stages using a technique developed by Kiriakouli and Negrepontis [KN]. The method consists of a double induction. To prove that every $r$-tuple of ordinals, $\left(\alpha_{1}, \ldots, \alpha_{r}\right)$, has a certain property $(\mathrm{P})$ one first shows that if $\left(\alpha_{1}, \ldots, \alpha_{r}\right)$ has $(\mathrm{P})$, then so does $\left(\alpha, \alpha_{1}, \ldots, \alpha_{r}\right)$. Next one demonstrates that if $\left(\alpha, \ldots, k, \alpha, \alpha_{1}, \ldots, \alpha_{r}\right)$ has the property for every $k \geq 1$, then so does $\left(\alpha+1, \alpha_{1}, \ldots, \alpha_{r}\right)$. The rest of the proof usually follows easily from these two results. In our case the key to proving Proposition 3.2 is the following lemma:

Lemma 3.3. Let $r \geq 1$ and let $0 \leq \alpha_{1} \leq \ldots \leq \alpha_{r}<\omega_{1}$. If $\left(\alpha_{1}, \ldots, \alpha_{r}\right)$ has the Dichotomy Property (D), then so does $\left(0, \alpha_{1}, \ldots, \alpha_{r}\right)$.

Proof. Let $\mathcal{F} \subseteq[\mathbb{N}]^{<\omega}$ be hereditary and $\bar{N}=\left(\bar{n}_{i}\right)_{i=1}^{\infty} \in[\mathbb{N}]$. Then we seek $L \in[\bar{N}]$ such that either $\left(\mathcal{S}_{0}, \mathcal{S}_{\alpha_{1}}, \ldots, \mathcal{S}_{\alpha_{r}}\right)(L) \subseteq \mathcal{F}$ or $\mathcal{N}$ has a winning strategy for the $\left(0, \alpha_{1}, \ldots, \alpha_{r}\right)$-game on $\mathcal{F}[L]$. We cannot find $L$ all at once; instead we must choose it bit by bit. We construct sequences $M_{l}=\left(m_{i}^{l}\right)_{i=1}^{\infty}$ with $\bar{N}=M_{0} \supseteq M_{1} \supseteq \ldots$ such that either $\left\{m_{l}^{l}\right\} \cup F \in \mathcal{F}$ for each $F \subseteq \mathcal{S}\left(M_{l}\right)$ with $F>m_{l}^{l}$, or else $\mathcal{N}$ has a winning strategy in the $\left(0, \alpha_{1}, \ldots, \alpha_{r}\right)$-game on $\mathcal{F}\left[M_{l}\right]$ provided the first choice of $\mathcal{S}$ is $\left\{m_{l}^{l}\right\}$. We may then choose $L$ as a diagonal subsequence of these sequences $M_{l}$.

We begin by defining

$$
\mathcal{F}_{1}=\left\{F:\left\{\bar{n}_{1}\right\} \cup F \in \mathcal{F}\left[M_{0}\right]\right\} .
$$

Since $\left(\alpha_{1}, \ldots, \alpha_{r}\right)$ has (D), it follows that there exists $\bar{M}_{1}=\left(\bar{m}_{i}^{1}\right)_{i=1}^{\infty} \in[\bar{N}]$ such that either $\mathcal{N}$ has a winning strategy for the $\left(\alpha_{1}, \ldots, \alpha_{r}\right)$-Schreier game on $\mathcal{F}_{1}\left[\bar{M}_{1}\right]$, or $\left(\mathcal{S}_{\alpha_{1}}, \ldots, \mathcal{S}_{\alpha_{r}}\right)\left(\bar{M}_{1}\right)$ is a subset of $\mathcal{F}_{1}$. Let $M_{1}=$ $\left(\bar{n}_{1}, \bar{m}_{2}^{1}, \bar{m}_{3}^{1}, \ldots\right)$ be the sequence $\bar{M}_{1}$ with its first element replaced by $\bar{n}_{1}$. Now, either $\left\{\bar{n}_{1}\right\} \cup E \in \mathcal{F}$ for each $E \in\left(\mathcal{S}_{\alpha_{1}}, \ldots, \mathcal{S}_{\alpha_{n}}\right)\left(M_{1}\right)$ with $E>\bar{n}_{1}$ or $\left\{\bar{n}_{1}\right\} \cup F \notin \mathcal{F}$ for every set $F \subseteq M_{1} \backslash\left\{\bar{n}_{1}\right\}$ resulting from $\mathcal{N}$ playing a winning strategy for the $\left(\alpha_{1}, \ldots, \alpha_{r}\right)$-game on $\mathcal{F}_{1}\left[M_{1}\right]$. This last follows since if $F \in \mathcal{F}_{1}\left[M_{1}\right]$ and $F>\bar{n}_{1}$, then $F \in \mathcal{F}_{1}\left[\bar{M}_{1}\right]$.

Suppose we have chosen sequences $\vec{N} \supseteq M_{1} \supseteq M_{2} \supseteq \ldots \supseteq M_{l-1}$ with the properties:

- If $M_{i}=\left(m_{j}^{i}\right)_{j=1}^{\infty}$ for $1 \leq i<l$, then $m_{j}^{i-1}=m_{j}^{i}$ whenever $1 \leq j \leq i$ and $1<i<l$. 
- For each $i=1, \ldots, l-1$, either $\left\{m_{i}^{i}\right\} \cup F \in \mathcal{F}$ for all $F \in\left(\mathcal{S}_{\alpha_{1}}, \ldots\right.$ $\left.\ldots, \mathcal{S}_{\alpha_{T}}\right)\left(M_{i}\right)$ with $F>m_{i}^{i}$, or else $\left\{m_{i}^{i}\right\} \cup F \notin \mathcal{F}$ for any $F \subseteq\left(m_{j}^{i}\right)_{j=i+1}^{\infty}$ resulting from $\mathcal{N}$ playing a winning strategy in the $\left(\alpha_{1}, \ldots, \alpha_{r}\right)$-game on $\mathcal{F}_{i}\left[M_{i}\right]$, where $\mathcal{F}_{i}=\left\{F:\left\{m_{i}^{i-1}\right\} \cup F \in \mathcal{F}\left[M_{i-1}\right]\right\}$.

To construct the next sequence $M_{l}$ we define

$$
\mathcal{F}_{l}=\left\{F:\left\{m_{l}^{l-1}\right\} \cup F \in \mathcal{F}\left[M_{l-1}\right]\right\} .
$$

Since $\left(\alpha_{1}, \ldots, \alpha_{r}\right)$ has $(\mathrm{D})$, it follows that there exists $\bar{M}_{l}=\left(\bar{m}_{i}^{l}\right)_{i} \in\left[M_{l-1}\right]$ such that either $\mathcal{N}$ has a winning strategy for the $\left(\alpha_{1}, \ldots, \alpha_{r}\right)$-Schreier game on $\mathcal{F}_{l}\left[\bar{M}_{l}\right]$, or $\left(\mathcal{S}_{\alpha_{1}}, \ldots, \mathcal{S}_{\alpha_{r}}\right)\left(\bar{M}_{l}\right) \subseteq \mathcal{F}_{l}$. Let

$$
M_{l}=\left(m_{1}^{l-1}, \ldots, m_{l}^{l-1}, \bar{m}_{l+1}^{l}, \bar{m}_{l+2}^{l}, \ldots\right)
$$

be the sequence $\bar{M}_{l}$ with the first $l$ elements replaced by the first $l$ elements of $M_{l-1}$. As with $M_{1}$ and $\mathcal{F}_{1}$, either $\left\{m_{l}^{l}\right\} \cup F^{\prime} \in \mathcal{F}$ for each $F \in$ $\left(\mathcal{S}_{\alpha_{1}}, \ldots, \mathcal{S}_{\alpha_{r}}\right)\left(M_{l}\right)$ with $F>m_{l}^{l}$, or $\left\{m_{l}^{l}\right\} \cup F \notin \mathcal{F}$ for every $F \subseteq\left(m_{i}^{l}\right)_{i>l}$ resulting from $\mathcal{N}$ playing a winning strategy for the $\left(\alpha_{1}, \ldots, \alpha_{r}\right)$-game on $\mathcal{F}_{l}\left[M_{l}\right]$.

We repeat this process for each $l \geq 1$. Let $M=\left(m_{k}\right)_{k=1}^{\infty}$ be the sequence defined by $m_{k}=m_{k}^{k}$ for each $k \geq 1$. Then for each $l \geq 1$, either $\left\{m_{l}\right\} \cup F \in \mathcal{F}$ for all $F \in\left(\mathcal{S}_{\alpha_{1}}, \ldots, \mathcal{S}_{\alpha_{r}}\right)(M)$ with $F>m_{l}$, or $\left\{m_{l}\right\} \cup F \notin \mathcal{F}$ for each $F \subseteq\left(m_{k}\right)_{k>l}$ resulting from $\mathcal{N}$ playing a winning strategy for the $\left(\alpha_{1}, \ldots, \alpha_{r}\right)$-game on $\mathcal{F}_{l}[M]$. This induces a coloring on $\mathbb{N}$; in the first case we color $l \in \mathbb{N}$ red, and in the second, blue.

Now, either there exists an infinite subsequence $J \in[\mathbb{N}]$ such that every $j \in J$ is colored red, in which case let $L=\left(m_{j}\right)_{j \in J}$, or there exists $k \geq 1$. such that $l$ is colored blue for all $l \geq k$, and then let $L=\left(m_{l}\right)_{l \geq k}$. In the first case it is clear that $\left(\mathcal{S}_{0}, \mathcal{S}_{\alpha_{1}}, \ldots, \mathcal{S}_{\alpha_{r}}\right)(L) \subseteq \mathcal{F}$. In the second case, if $\mathcal{S}$ picks $\{n\}$ with $n \notin L$, then the resulting set cannot be in $\mathcal{F}[L]$. Otherwise $\mathcal{S}$ picks $\left\{m_{l}\right\}$ for some $l \geq k$ and then $\mathcal{N}$ has a winning strategy for the $\left(\alpha_{1}, \ldots, \alpha_{r}\right)$-Schreier game on $\mathcal{F}_{l}\left[\left(m_{i}\right)_{i>l}\right]$. In either situation we see that $\mathcal{N}$ has a winning strategy for the $\left(0, \alpha_{1}, \ldots, \alpha_{r}\right)$-Schreier game on $\mathcal{F}[L]$ as required.

LEMMA 3.4. If $0 \leq \alpha<\alpha_{1} \leq \ldots \leq \alpha_{r}$, for some $r \geq 0$, and the $(k+r)$-tuple $\left(\alpha, . k^{k}, \alpha, \alpha_{1}, \ldots, \alpha_{r}\right)$ has property (D) for every $k \geq 1$, then $\left(\alpha+1, \alpha_{1}, \ldots, \alpha_{r}\right)$ has property (D).

Proof. Let $\mathcal{F} \subseteq[\mathbb{N}]^{<\omega}$ be hereditary, let $\bar{N} \in[\mathbb{N}]$ and find sequences $\bar{N} \supseteq$ $L_{1} \supseteq L_{2} \supseteq \ldots$ such that for each $k$ either $\left(\mathcal{S}_{\alpha}, . . ., \mathcal{S}_{\alpha}, \mathcal{S}_{\alpha_{1}}, \ldots, \mathcal{S}_{\alpha_{r}}\right)\left(L_{k}\right)$ $\subseteq \mathcal{F}$, or $\mathcal{N}$ has a winning strategy in the $\left(\alpha, \ldots, \alpha, \alpha_{1}, \ldots, \alpha_{r}\right)$-Schreier game on $\mathcal{F}\left[L_{k}\right]$. In this last case $\mathcal{N}$ has a winning strategy in the $(\alpha+1$, $\left.\alpha_{1}, \ldots, \alpha_{r}\right)$-Schreier game on $\mathcal{F}\left[L_{k}\right]$ given as follows: for the $(\alpha+1)$-game $\mathcal{N}$ picks $k$ and then plays a winning strategy in the $\left(\alpha, k ., \alpha, \alpha_{1}, \ldots, \alpha_{r}\right)$ -
Schreier game on $\mathcal{F}\left[L_{k}\right]$. Otherwise we set $L=\left(l_{k}^{k}\right)$ and then we obtain $\left(\mathcal{S}_{\alpha+1}, \mathcal{S}_{\alpha_{1}}, \ldots, \mathcal{S}_{\alpha_{r}}\right)(L) \subseteq \mathcal{F}$.

LEMMA 3.5. If $\alpha$ is a limit ordinal with $\alpha \leq \alpha_{1} \leq \ldots \leq \alpha_{r}$, for some $r \geq 0$, and $\left(\beta, \alpha_{1}, \ldots, \alpha_{r}\right)$ has property (D) for every $\beta<\alpha$, then $\left(\alpha, \alpha_{1}, \ldots, \alpha_{r}\right)$ has property (D).

Proof. As before, let $\mathcal{F} \subseteq[\mathbb{N}]<\omega$ be hereditary, let $\bar{N} \in[\mathbb{N}]$ and find $\bar{N} \supseteq L_{1} \supseteq L_{2} \supseteq \ldots$ such that for every $k$ either $\left(\mathcal{S}_{\beta_{k}}, \mathcal{S}_{\alpha_{1}}, \ldots, \mathcal{S}_{\alpha_{r}}\right)\left(L_{k}\right) \subseteq \mathcal{F}$, or $\mathcal{N}$ has a winning strategy in the $\left(\beta_{k}, \alpha_{1}, \ldots, \alpha_{r}\right)$-Schreier game on $\mathcal{F}\left[L_{k}\right]$, where $\beta_{k} / \alpha$ is the sequence used to define $\mathcal{S}_{\alpha}$. In the second case $\mathcal{N}$ has a winning strategy in the $\left(\alpha, \alpha_{1}, \ldots, \alpha_{r}\right)$-game on $\mathcal{F}\left[L_{k}\right]$ by choosing $k$ and playing a winning strategy in the $\left(\beta_{k}, \alpha_{1}, \ldots, \alpha_{r}\right)$-Schreier game on $\mathcal{F}\left[L_{k}\right]$. Otherwise we let $L=\left(l_{k}^{k}\right)$, and then $\left(\mathcal{S}_{\alpha}, \mathcal{S}_{\alpha_{1}}, \ldots, \mathcal{S}_{\alpha_{r}}\right)(L) \subseteq \mathcal{F}$.

LEMMA 3.6. If $0 \leq \alpha_{1} \leq \ldots \leq \alpha_{r}$, for some $r \geq 1$, and $\left(\alpha_{1}, \ldots, \alpha_{r}\right)$ has property (D) then so too does $\left(\beta_{1}, \ldots, \beta_{s}, \alpha_{1}, \ldots, \alpha_{r}\right)$ for all $\beta_{1} \leq \ldots \leq$ $\beta_{s} \leq \alpha_{1}$ and each $s \geq 1$.

Proof. We prove this by induction on $\beta_{s}$ for arbitrary $s$ and $\alpha_{1} \geq \beta_{s}$. When $\beta_{s}=0$ the result follows by iterating Lemma $3.3 s$ times.

Suppose that we have proven the result for $\beta=\beta_{s}$, i.e. we have shown that for every $\alpha_{r} \geq \ldots \geq \alpha_{1} \geq \beta$, if $\left(\alpha_{1}, \ldots, \alpha_{r}\right)$ has property (D), then so too does $\left(\gamma_{1}, \ldots, \gamma_{k}, \beta, \alpha_{1}, \ldots, \alpha_{r}\right)$ for all $\gamma_{1} \leq \ldots \leq \gamma_{k} \leq \beta$. Clearly, we may take each $\gamma_{i}=\beta$ and so in particular we have proven that for every $\alpha_{r} \geq \ldots$ $\ldots \geq \alpha_{1}>\beta$, if $\left(\alpha_{1}, \ldots, \alpha_{r}\right)$ has property (D), then $\left(\beta, \ldots, \beta, \alpha_{1}, \ldots, \alpha_{r}\right)$ also has property (D) for each $k \geq 1$. Hence by Lernma 4.3 so does $(\beta+1$, $\left.\alpha_{1}, \ldots, \alpha_{r}\right)$. Iterating this argument, we see that if $\alpha_{r} \geq \ldots \geq \alpha_{1}>\beta$ and $\left(\alpha_{1}, \ldots, \alpha_{r}\right)$ has property (D), then $\left(\beta+1, ., ., \beta+1, \alpha_{1}, \ldots, \alpha_{r}\right)$ also has property (D) for all $l \geq 1$, and hence $\left(\gamma_{1}, \ldots, \gamma_{k}, \beta+1, .,, \beta+1, \alpha_{1}, \ldots, \alpha_{r}\right)$ does too, for all $\gamma_{1} \leq \ldots \leq \gamma_{k} \leq \beta$, by the result for $\beta$. In other words, for every $\alpha_{r} \geq \ldots \geq \alpha_{1} \geq \beta+1$, if $\left(\alpha_{1}, \ldots, \alpha_{r}\right)$ has property (D), then so too does $\left(\beta_{1}, \ldots, \beta_{s}, \alpha_{1}, \ldots, \alpha_{r}\right)$ for all $\beta_{1} \leq \ldots \leq \beta_{s} \leq \beta+1$ and any $s \geq 1$, as required.

If $\beta_{s}$ is a limit ordinal and we have proven the result for each $\beta<\beta_{s}$, then $\left(\beta, \alpha_{1}, \ldots, \alpha_{r}\right)$ has property (D) for each $\beta<\beta_{s}$. Thus, by Lemma 3.5, the $(r+1)$-tuple $\left(\beta_{s}, \alpha_{1}, \ldots, \alpha_{r}\right)$ also has property (D). Now, as in the successor case, we conclude that $\left(\beta_{1}, \ldots, \beta_{s}, \alpha_{1}, \ldots, \alpha_{r}\right)$ has property (D) as required.

Proof of Proposition 3.2. We prove by induction that $(\alpha)$ has property (D) for each $\alpha<\omega_{1}$, and then the result follows from Lemma 3.6. Let $\alpha=0$, let $\mathcal{F} \subseteq \mathbb{N}]^{<\omega}$ be hereditary and let $\bar{N} \in[\mathbb{N}]$. Let $L=\{n \in \bar{N}:\{n\} \in \mathcal{F}\}$; if $L$ is infinite let $M=L$, and then $\mathcal{S}_{0}(M) \subseteq \mathcal{F}$. Otherwise let $M=\bar{N} \backslash L$, 
then $\mathcal{N}$ has a winning strategy for the 0 -Schreier game on $\mathcal{F}[M]$ since this set is empty. This completes the proof for $\alpha=0$.

If $(\alpha)$ has (D) then by Lemma 3.6 so does $(\alpha, . k, \alpha)$ for each $k \geq 1$. Thus $(\alpha+1)$ has (D) by Lemma 3.4. If $\alpha$ is a limit ordinal and $(\beta)$ has (D) for each $\beta<\alpha$ then $(\alpha)$ has (D) by Lemma 3.5.

4. The main result. In this section we prove Theorem 1.1. Actually we prove a somewhat stronger statement:

THEOREM 4.1. For all $r \geq 1$ and each $r$-tuple of countable ordinals $0 \leq \alpha_{1} \leq \ldots \leq \alpha_{r}<\omega_{1}$, if $\mathcal{F}$ is a hereditary collection, $\mathcal{F} \subseteq[\mathbb{N}]<\omega$ and $\bar{N} \in[\mathbb{N}]$, then either there exists $M \in[\bar{N}]$ such that $\left(\mathcal{S}_{\alpha_{1}}, \ldots, \mathcal{S}_{\alpha_{r}}\right)(M) \subseteq \mathcal{F}$, or there exist $M \in[\bar{N}], N \in[\mathbb{N}]$ such that $\mathcal{F}[M](N) \subseteq\left(\mathcal{S}_{\alpha_{1}}, \ldots, \mathcal{S}_{\alpha_{r}}\right)$.

Proposition 4.2. If $\mathcal{S}$ and $\mathcal{N}$ play a bound $\left(\alpha_{1}, \ldots, \alpha_{r}\right)$-game, then there exists $N=\left(n_{i}\right) \in[\mathbb{N}]$ such that if $E$ is any result of this bound game where $\mathcal{S}$ chose $E$ as small as possible, then $n_{E}=\left\{n_{i}: i \in E\right\} \in$ $\left(\mathcal{S}_{\alpha_{1}}, \ldots, \mathcal{S}_{\alpha_{r}}\right)$.

Before we give the proof of this proposition we recall the notion of spreading. A collection $\mathcal{F} \subseteq[\mathbb{N}]^{<\omega}$ is spreading if it has the property that if $G=\left\{g_{1}, \ldots, g_{n}\right\} \in \mathcal{F}$ and $H=\left\{h_{1}, \ldots, h_{n}\right\}$ satisfies $g_{j} \leq h_{j}(j=1, \ldots, n)$, then also $H \in \mathcal{F}$. In this case we say that $H$ is a spreading of $G$. Moreover, it is easy to see that if $\mathcal{F}$ is spreading and $M=\left(m_{i}\right), N=\left(n_{i}\right) \in[\mathbb{N}]$ satisfy $m_{i} \leq n_{i}$ for all $i$, then $m_{E} \in \mathcal{F}$ implies that $n_{E} \in \mathcal{F}$

Proof of Proposition 4.2. We first prove the result for $r=1$ by induction on $\alpha$. This is then easy to generalize. In order to find the sequence $N=\left(n_{t}\right)$ we construct an increasing function $f: \mathbb{N} \rightarrow \mathbb{N}$ and let $n_{t}=f(t)$.

CASE 1: $\alpha=0$. This is clearly true, just by setting $f(t)=t$.

CASE 2: $\alpha \Rightarrow \alpha+1$. We assume that for any bound $\alpha$-game there exists a function $f$ as above. Now, an $(\alpha+1)$-game consists of $\mathcal{N}$ choosing $k$ and then the two players playing a bound $(\alpha, k, \alpha)$-game. Since the $(\alpha+1)$-game is bound there is only one choice of $k$ which $\mathcal{N}$ may make. For each of the bound $\alpha$-games which make up the $(\alpha+1)$-game we shall choose below a function $f^{i}$ such that $f^{i}\left(E_{i}\right)=\left\{f^{i}(t): t \in E_{i}\right\} \in \mathcal{S}_{\alpha}$, for any set $E_{i}$ resulting from the $i$ th $\alpha$-game. We then let $f(t)=k+\sum_{i=1}^{k} f^{i}(t)$.

The first $\alpha$-game is already fixed, so we may choose $f^{1}$ using the hypothesis. However, the $i$ th $\alpha$-game, while bound, depends on which sets were picked in the first $i-1$ games, so we cannot just pick $f^{i}$ straight from the hypothesis-instead we have to cover all possible bound $\alpha$-games which may be played. Fortunately, for any fixed integer $t$ only finitely many bound $(\alpha, \stackrel{i}{-1}, \alpha)$-games can be played which finish before $t$; let this number be $s$ Thus there are $s$ possible bound $\alpha$-games we could be playing. If $s \neq 0$, then let the functions from these be $f_{1}^{i}, \ldots, f_{s}^{i}$, and let $f^{i}(t)=\sum_{j=1}^{s} f_{j}^{i}(t)$. Otherwise let $f^{i}(t)=t$.

We must now show that the function $f$ given by $f(t)=k+\sum_{i=1}^{k} f^{i}(t)$ is the function we seek for the bound $(\alpha+1)$-game. Let $E=\bigcup_{i=1}^{k} E_{i}$ be the result of the bound $(\alpha, k, \alpha)$-game where $\mathcal{S}$ chose $E$ as small as possible and where $E_{i}$ is the result of the $i$ th $\alpha$-game. We show that $f^{i}\left(E_{i}\right) \in \mathcal{S}_{\alpha}$ for $i \leq k$. We already know that this works for $i=1$ by the hypothesis. Then for $1<i \leq k$, once we have chosen $E_{1}<\ldots<E_{i-1}$ we will have fixed the bound $\alpha$-game we are playing when choosing $E_{i}$. Let the function for this game be $f^{\prime}$; then from the induction hypothesis, $f^{\prime}\left(E_{i}\right)=\left\{f^{\prime}(t): t \in E_{i}\right\} \in \mathcal{S}_{\alpha}$. But by the construction of $f^{i}$ we know that $f^{i}(t) \geq f^{\prime}(t)$ for each $t$ in $E_{i}$. To obtain $f^{i}\left(E_{i}\right) \in \mathcal{S}_{\alpha}$ recall that the collection $\mathcal{S}_{\alpha}$ is spreading and clearly $f^{i}\left(E_{i}\right)$ is a spreading of $f^{\prime}\left(E_{i}\right)$, hence $f^{i}\left(E_{i}\right)$ is also in $\mathcal{S}_{\alpha}$. Finally, since $f(t) \geq f^{i}(t)$ for every $i$ and $t$, it follows that $f\left(E_{i}\right) \in \mathcal{S}_{\alpha}(i=1, \ldots, k)$ and since $f(1) \geq k$ we have $k \leq E_{1}<\ldots<E_{k}$, so that $E=\bigcup_{i=1}^{k} E_{i} \in \mathcal{S}_{\alpha+1}$ as required.

CASE 3: $\alpha$ is a limit ordinal. For the $\alpha$-game $\mathcal{N}$ is bound to pick $l$ and then they play a bound $\alpha_{l}$-game (where $\alpha_{n} / \alpha$ is the sequence of ordinals increasing to $\alpha$ fixed in the definition of $\mathcal{S}_{\alpha}$ ). By assumption we may choose $f^{\prime}$ for the $\alpha_{l}$-game such that if $E$ is the result of the $\alpha_{l}$-game where $\mathcal{S}$ chose $E$ as small as possible, then $f^{\prime}(E) \in \mathcal{S}_{\alpha_{l}}$. Let $f(t)=f^{\prime}(t)+l$. Now, $f^{\prime}(E) \in \mathcal{S}_{\alpha_{l}}$ for the $E$ we fixed initially, which implies $f(E) \in \mathcal{S}_{\alpha_{l}}$, since $f(E)$ is a spreading of $f^{\prime}(E)$. Finally, $f(1) \geq l$, hence $f(E) \in \mathcal{S}_{\alpha}$ since $\left\{F \in \mathcal{S}_{\alpha_{l}}: l \leq F\right\} \subseteq \mathcal{S}_{\alpha}$.

To generalize for $\left(\alpha_{1}, \ldots, \alpha_{r}\right)$ we proceed as in Case 2 , using bound $\alpha_{i}$-games $(i=1, \ldots, r)$.

COROLLARY 4.3. If $\mathcal{N}$ has a winning strategy for an $\left(\alpha_{1}, \ldots, \alpha_{r}\right)$-Schreier game on a hereditary collection $\mathcal{F} \subseteq[\mathbb{N}]^{<\omega}$, then there exists $N=\left(n_{i}\right) \in[\mathbb{N}]$ such that $\mathcal{F}(N) \subseteq\left(\mathcal{S}_{\alpha_{1}}, \ldots, \mathcal{S}_{\alpha_{\tau}}\right)$.

P r o of. Suppose $\mathcal{N}$ has a winning strategy for an $\left(\alpha_{1}, \ldots, \alpha_{r}\right)$-Schreier game on $\mathcal{F} \subseteq[\mathbb{N}]^{<\omega}$. Let $\mathcal{N}, \mathcal{S}$ play the bound $\left(\alpha_{1}, \ldots, \alpha_{r}\right)$-game where $\mathcal{N}$ always chooses $l$ as small as possible so that $\mathcal{N}$ will win. Let $E \in \mathcal{F}$; then we may decompose $E=\bigcup_{i=1}^{p} E_{i}$ according to this game as follows. If the first set which $\mathcal{S}$ chooses must have length greater than or equal to $l_{1}$, then let $E_{1}=\left\{e_{1}, \ldots, e_{l_{1}}\right\}$; if $\mathcal{S}$ has chosen $E_{1}<\ldots<E_{q-1}$ and $\mathcal{S}$ must pick the $q$ th set to have length at least $l_{q}$, then let $E_{q}$ be the next $l_{q}$ elements of $E$ after $E_{q-1}$. Since $\mathcal{N}$ has a winning strategy, and $E \in \mathcal{F}$, this process must exhaust $E$, but at that point let $\mathcal{S}$ continue the game, always choosing sets as small as possible, and let $\bar{E}$ be the union of the sets obtained (including $E$ ). Now, by Proposition 4.2 , there exists $N=\left(n_{i}\right) \in[\mathbb{N}]$ such 
that $n_{F} \in\left(\mathcal{S}_{\alpha_{1}}, \ldots, \mathcal{S}_{\alpha_{r}}\right)$ for any set $F$ resulting from such a game. Thus $n_{\bar{E}} \in\left(\mathcal{S}_{\alpha_{1}}, \ldots, \mathcal{S}_{\alpha_{r}}\right)$, and hence so is $n_{E}$ since $E \subseteq \bar{E}$ and $\mathcal{S}_{\alpha}$ is hereditary for each $\alpha$. So finally, $\mathcal{F}(N) \subseteq\left(\mathcal{S}_{\alpha_{1}}, \ldots, \mathcal{S}_{\alpha_{r}}\right)$ as required.

These results are sufficient to prove Theorem 4.1 :

Proof of Theorem 4.1. Let $r \geq 1$, let $0 \leq \alpha_{1} \leq \ldots \leq \alpha_{r}<\omega_{1}$, and let $\mathcal{F}$ be a hereditary collection, $\mathcal{F} \subseteq[\mathbb{N}]<\omega$. Since $\left(\alpha_{1}, \ldots, \alpha_{r}\right)$ has the Dichotomy Property, it follows that either there exists $M \in[\bar{N}]$ such that $\left(\mathcal{S}_{\alpha_{1}}, \ldots, \mathcal{S}_{\alpha_{r}}\right)(M) \subseteq \mathcal{F}$, in which case the proof is complete, or there exists $M \in[\bar{N}]$ such that $\mathcal{N}$ has a winning strategy for the $\left(\alpha_{1}, \ldots, \alpha_{r}\right)$-Schreier game on $\mathcal{F}[M]$. Now $\mathcal{F}[M]$ is again hereditary and thus by Corollary 4.3 there exists $N \in[\mathbb{N}]$ such that $\mathcal{F}[M](N) \subseteq\left(\mathcal{S}_{\alpha_{1}}, \ldots, \mathcal{S}_{\alpha_{r}}\right)$ as required.

Theorem 1.1 follows from Theorem 4.1 as an immediate corollary.

REMARK 4.4. It should be noted that Theorem 4.1 is no longer true if we do not first restrict $\mathcal{F}$ to a subsequence of $\mathbb{N}$. Indeed, we have the following example:

EXAMPLE 4.5. We construct a hereditary collection $\mathcal{F} \subseteq[\mathbb{N}]<\omega$ such that for every $M \in[\mathbb{N}]$ we have both $\mathcal{S}_{1}(M) \nsubseteq \mathcal{F}$ and $\mathcal{F}(M) \nsubseteq \mathcal{S}_{1}$.

Let $F_{k}=\left\{2^{k}+1, \ldots, 2^{k}+k\right\}$ for each $k \geq 1$ and let

$$
\mathcal{F}=\bigcup_{k=1}^{\infty}\left\{\{1\} \cup E, E: E \subseteq F_{k}\right\}
$$

Then $\mathcal{F}$ is clearly hereditary. Let $M \in[\mathbb{N}]$ and let $l=m_{1}$. Then $F=$ $\left\{m_{1}\right\} \cup m_{F_{l}} \in \mathcal{F}(M)$, but $|F|=l+1>\min F$ and hence $F \notin \mathcal{S}_{1}$. Furthermore, $\mathcal{S}_{1}(M) \nsubseteq \mathcal{F}$; indeed, suppose $E \in \mathcal{S}_{1}(M)$ and $E \in \mathcal{F}$ with $|E|>2$. Let $E=\left\{e_{1}, \ldots, e_{p}\right\}$ and find $k$ such that $E<F_{k}$. Now let $E^{\prime}=\left\{e_{1}, \ldots, e_{p-1}, m_{2^{k}+1}\right\}$. Then $E^{\prime}$ is still in $\mathcal{S}_{1}(M)$ since this collection is spreading, but $E^{\prime} \notin \mathcal{F}$ because if $F, F^{\prime} \in \mathcal{F}$ then either $F \subseteq F^{\prime}, F^{\prime} \subseteq F$ or $\left|F \cap F^{\prime}\right|=0$ or 1 . None of these is true for $E, E^{\prime}$

5. Application. In this section we use Theorem 1.1 to provide an alternative proof of a result in a paper of Argyros, Mercourakis and Tsarpalias [AMT]. We first state some definitions.

DEFINITION 5.1. Let $\mathcal{F}$ be an adequate family (hereditary and closed) of finite subsets of $\mathbb{N}$ as defined in Section 2. For $L \in[\mathbb{N}]$ we define the strong Cantor-Bendixson derivative of $\mathcal{F}[L]$ for each ordinal $\alpha<\omega_{1}$ to be

$\mathcal{F}[L]^{(1)}=\{A \in \mathcal{F}[L]: A$ is a cluster point of $\mathcal{F}[A \cup N]$ for each $N \in[L]\}$.

Thus for finite $A \subseteq L$ we have $A \in \mathcal{F}[L]^{(1)}$ if and only if $\{l \in L: A \cup\{l\} \notin \mathcal{F}\}$

is finite. If we have defined $\mathcal{F}[L]^{(\alpha)}$, the $\alpha$ th strong Cantor-Bendixson deriva- tive of $\mathcal{F}[L]$, then we define the $(\alpha+1)$ th derivative as follows:

$$
\mathcal{F}[L]^{(\alpha+1)}=\left(\mathcal{F}[L]^{(\alpha)}\right)[L]^{(1)} .
$$

If $\alpha$ is a limit ordinal and we have defined $\mathcal{F}[L]^{(\beta)}$ for each $\beta<\alpha$, then we set

$$
\mathcal{F}[L]^{(\alpha)}=\bigcap_{\beta<\alpha} \mathcal{F}[L]^{(\beta)} .
$$

The strong Cantor-Bendixson index [AMT] of $\mathcal{F}[L]$ is defined to be the smallest countable ordinal $\alpha<\omega_{1}$ such that $\mathcal{F}[L]^{(\alpha)}=\emptyset$. We denote this index by $s(\mathcal{F}[L])$. For more details concerning the strong Cantor-Bendixson derivative and index refer to [AMT].

REMARK 5.2. The following are stated in [AMT] or are simple consequences of that work:

(i) The strong Cantor-Bendixson index must be a successor ordinal

(ii) For each $\alpha<\omega_{1}$ we have $s\left(\mathcal{S}_{\alpha}\right)=\omega^{\alpha}+1$.

(iii) If $\mathcal{F} \subseteq[\mathbb{N}]<\omega$ is spreading, then $s(\mathcal{F}[L])=s(\mathcal{F})$ for every $L \in[\mathbb{N}]$.

(iv) If $s(\mathcal{F}[L])>\alpha$, then $s(\mathcal{F}[M])>\alpha$ for every $M \in[L]$ ([AMT], Proposition 2.2.3).

We prove the following result from [AMT] (Theorem 2.2.6):

THEOREM 5.3 ([AMT] $)$. Let $\mathcal{F} \subseteq[\mathbb{N}]^{<\omega}$ be an adequate family. If there exists $L \in[\mathbb{N}]$ such that $s(\mathcal{F}[L])>\omega^{\alpha}$, then there exists $M \in[L]$ such that $\mathcal{S}_{\alpha}(M) \subseteq \mathcal{F}[M]$

Proof. Let $\mathcal{F} \subseteq[\mathbb{N}]^{<\omega}$ be an adequate family and let $L \in[\mathbb{N}]$ satisfy $s(\mathcal{F}[L])>\omega^{\alpha}$. Suppose first that in fact $s(\mathcal{F}[L])>\omega^{\alpha}+1$. Now, by Theorem 1.1, either there exists $M \in[L]$ such that $\mathcal{S}_{\alpha}(M) \subseteq \mathcal{F}[M]$ as required, or else there exist $M \in[L], N \in[\mathbb{N}]$ such that $\mathcal{F}[M](N) \subseteq \mathcal{S}_{\alpha}$. We can easily see that the index of $\mathcal{F}[M]$ is the same as the index of $(\mathcal{F}[M](N))\left[N_{M}\right]$ where $N_{M}=\left(n_{m}\right)_{m \in M}$. Indeed, if $\left(A_{i}\right)$ is a sequence in $\mathcal{F}[M]^{(\beta)}$ converging to $A \in \mathcal{F}[M]^{(\beta+1)}$, then $\left(n_{A_{i}}\right)$ is a sequence in $(\mathcal{F}[M](N))\left[N_{M}\right]^{(\beta)}$ converging to $n_{A} \in(\mathcal{F}[M](N))\left[N_{M}\right]^{(\beta+1)}$ and vice versa. Thus, if $\mathcal{F}[M](N) \subseteq \mathcal{S}_{\alpha}$, then

$$
s(\mathcal{F}[M])=s\left((\mathcal{F}[M](N))\left[N_{M}\right]\right) \leq s\left(\mathcal{S}_{\alpha}\left[N_{M}\right]\right)=\omega^{\alpha}+1
$$

However, by Remark 5.2(iv), $s(\mathcal{F}[M])>\omega^{\alpha}+1$, a contradiction. Thus the second case above cannot happen.

To finish the proof we assume that $s(\mathcal{F}[L])=\omega^{\alpha}+1$ and define

$$
\overline{\mathcal{F}}=\{\{n\} \cup F: F \in \mathcal{F}, n<F\} \cup \mathcal{F} .
$$

If $A \in \mathcal{F}[L]^{(\beta)} \backslash\{\emptyset\}$ and $l \in L$ with $l<A$, then $\{l\} \cup A \in \overline{\mathcal{F}}[L]^{(\beta)}$. Indeed, suppose this is true for some ordinal $\beta<\alpha$ and let $A \in \mathcal{F}[L]^{(\beta+1)} \backslash\{\emptyset\}$ and $l \in L$ with $l<A$. Then there exists a sequence $\left(A_{i}\right) \subseteq \mathcal{F}[L]^{(\beta)}$ converging to 
$A$. Now, $\left(\{l\} \cup A_{i}\right)$ is a sequence in $\overline{\mathcal{F}}[L]^{(\beta)}$ converging to $\{l\} \cup A$, hence $\{l\} \cup$ $A \in \overline{\mathcal{F}}[L]^{(\beta+1)}$ as required. The limit ordinal case is clear. Since $\mathcal{F}[L]^{\left(\omega^{\alpha}\right)} \neq \emptyset$, it follows that $\mathcal{F}[L]^{(\beta)}$ is infinite for each $\beta<\omega^{\alpha}$, so that $\{l\} \in \overline{\mathcal{F}}[L]^{(\beta)}$ for every $l \in L$, and each $\beta<\omega^{\alpha}$. Thus $\{l\} \in \overline{\mathcal{F}}[L]^{\left(\omega^{\alpha}\right)}$ for every $l \in L$ and hence $\emptyset \in \overline{\mathcal{F}}[L]^{\left(\omega^{\alpha}+1\right)}$, so that $s(\overline{\mathcal{F}}[L])>\omega^{\alpha}+1$. Finally, we apply the previous case to $\overline{\mathcal{F}}[L]$ to obtain $M=\left(m_{i}\right) \in[L]$ with $\mathcal{S}_{\alpha}(M) \subseteq \overline{\mathcal{F}}[M]$. Then setting $M^{\prime}=\left(m_{i}\right)_{i>2}$ we have $\mathcal{S}_{\alpha}\left(M^{\prime}\right) \subseteq \mathcal{F}\left[M^{\prime}\right]$ as required.

\section{On Mackey topology for groups}

by

\section{References}

[AA] D. Alspach and S. Argyros, Complexity of weakly null sequences, Dissertationes Math. 321 (1992).

[AO] D. Alspach and E. Odell, Averaging weakly null sequences, Lecture Notes in Math. 1332, Springer, 1988, 126-144

[AD] S. Argyros and I. Deliyanni, Examples of asymptotic $\ell_{1}$ Banach spaces, preprint.

[AMT] S. Argyros, S. Mercourakis and A. Tsarpalias, Convex unconditionality and summability of weakly null sequences, preprint.

[FJ] T. Figiel and W. B. Johnson, A uniformly convex Banach space which contains no $\ell_{p}$, Compositio Math. 29 (1974), 179-190.

[KN] P. Kiriakouli and S. Negrepontis, Baire-1 functions and spreading models of $\ell_{1}$, preprint.

[OTW] E. Odell, N. Tomczak-Jaegermann and R. Wagner, Proximity to $\ell_{1}$ and distortion in asymptotic $\ell_{1}$ spaces, preprint.

[Sch] J. Schreier, Gegenbeispiel zur Theorie der schwachen Konvergenz, Studia Math. 2 (1930), 58-62.

[T] B. S. Tsirelson, Not every Banach space contains $\ell_{p}$ or $c_{0}$, Functional Anal. Appl. 8 (1974), 138-141.

Department of Mathematics

Oklahoma State University

Stillwater, Oklahoma 74078-0613

U.S.A.

E-mail: rjudd@math.okstate.edu

M. J. CHASCO (Pamplona), E. MARTÍN-PEINADOR (Madrid) and V. TARIELADZE (Tbilisi)

Abstract. The present paper is a contribution to fill in a gap existing between the theory of topological vector spaces and that of topological abelian groups. Topological vector spaces have been extensively studied as part of Functional Analysis. It is natural to expect that some important and elegant theorems about topological vector spaces may have analogous versions for abelian topological groups. The main obstruction to get such versions is probably the lack of the notion of convexity in the framework of groups. However, the introduction of quasi-convex sets and locally quasi-convex groups by Vilenkin $[26]$ and the work of Banaszczyk [1] have paved the way to obtain theorems of this nature. We study here the group topologies compatible with a given duality. We have obtained, among others, the following result: for a complete metrizable topological abelian group, there always exists a finest locally quasi-convex topology with the same set of continuous characters as the original topology. We also give a description of this topology as an characters as the original topology. We also give a description of this topology as an
S-topology and we prove that, for the additive group of a complete metrizable topological vector space, it coincides with the ordinary Mackey topology.

Introduction. A vector topology $\tau$ in a real topological vector space $E$ is called a compatible topology for $E$ if the set of all $\tau$-continuous linear functionals is the same as the set $E^{*}$ of all continuous linear functionals in the original topology of $E$. The Mackey-Arens theorem implies that if $E$ is a topological vector space, then there exists a finest locally convex compatible topology for $E$, called in the literature the Mackey topology, and frequently denoted by $\tau\left(E, E^{*}\right)$. Similar assertions are also proved for topological vector spaces over non-Archimedian fields [14]. In the present paper we study the question for topological abelian groups.

1991 Mathematics Subject Classification: 46A20,43A40, 46A16.

Key words and phrases: locally convex space, Mackey topology, continuous character, weakly compact, locally quasi-convex group.

The second author supported by Ministerio de Educación, grant PB93-0454-C0201.

The third author partially supported by International Science Foundation, grant MXC 200. This paper was written at Universidad Complutense de Madrid, during two consecutive visits in the years 1995 and 1996. Thanks are due for hospitality. 